\title{
Macroscopical matter derived from Quantum Field Theory
}

\author{
G. Domenech* \\ and M. L. Levinas ${ }^{\dagger}$ \\ Instituto de Astronomía y Física del Espacio (IAFE) \\ Casilla de Correo 67, Sucursal 28, 1428 Buenos Aires, \\ Argentina
}

\begin{abstract}
We develop the WKB expansion to relate Quantum Field Theory variables with those describing macroscopical matter. We find that, up to the first quantum correction, free scalar fields correspond to perfect fluids with pressure. We also find the law of motion for the associated particles which takes into account deviations from the classical trajectories, showing that they do not follow unaccelerated straight line trajectories.
\end{abstract}

Keywords: Quantum Field Theory, Fluid dynamics

PACS number (s): 11.10.-z, 03.40.Gc

\section{INTRODUCTION}

The aim of this article is to find the macroscopical expression of the energy-momentum tensor of a free scalar field derived from Quantum Field Theory (QFT) up to the first quantum order in the WKB expansion, and to study its macroscopical behavior. In previous works we established the relation among QFT variables -amplitudes and phaseswith macroscopic fluid variables -proper energy and four-velocity- but considering only the lowest order in $\hbar$ in the scheme of general curved space-times, in Riemannian and non-Riemannian geometries. In both cases, free scalar matter corresponds to perfect fluids without pressure following geodesics[1, 2]. In fact, this statement also holds for flat Minkowski space-time where geodesics must be understood as straight non-accelerated trajectories (inertial principle). In the present article we prove that up to the following order, free scalar fields correspond to perfect fluids with pressure. We also find the corresponding law of motion which shows dispersions from inertial motion. Up to our knowledge, these are new results which give a deeper insight in the relation between second quantization and standard description of macroscopical matter. We believe that these results contribute to place QFT as the ultimate description of matter structure.

The paper is organized as follows: in section II we expand the energy-momentum tensor of the free scalar field. In section III, we find the relation between microscopical and macroscopical variables and prove that free scalar matter must be represented by a perfect fluid with pressure when quantum corrections are taken into account. In IV we find the equation which relates the WKB amplitudes that are involved in the corresponding definitions of proper energy densities and pressures; this section is also devoted to find the first quantum correction to the classical equations of motion. Finally, in V, we make a summary of our results.

\section{THE WKB EXPANSION OF THE ENERGY-MOMENTUM TENSOR}

In order to study the relationship between quantum and classical dynamics, we start considering the complex wave function $\Psi$ in terms of the path integral formalism. In the first quantization scheme, the wave function of the particle may be expressed as:

$$
\Psi\left(x_{o}, x_{f}\right)=N \int e^{i S_{c l}[x] / \hbar} \mathcal{D} x
$$

where $S_{c l}[x]$ is the classical action which is a functional of the trajectory $x(t)$ depending on the initial and final fixed data $\left(x_{o}, x_{f}\right)$ and $N$ is a normalization factor. If $S_{c l}$ is the action of a non-relativistic particle, then $\Psi$ corresponds to a

* Fellow of the Consejo Nacional de Investigaciones Cientificas y Tecnicas (CONICET) - levinas@iafe.uba.ar

${ }^{\dagger}$ Fellow of the Consejo Nacional de Investigaciones Cientificas y Tecnicas (CONICET) - levinas@iafe.uba.ar 
wave function which satisfies the Schroedinger equation [3]. On the other hand, when we work in the scheme of second quantization, $\Psi$ must be taken to be a field satisfying the relativistic field equation obtained for the corresponding representation of the Lorentz group. Field equations arise minimizing action $S[\Psi]$ with respect to the field:

$$
\frac{\delta S[\Psi]}{\delta \Psi}=0
$$

The expansion of eq. (1) in powers of $\hbar$ reads:

$$
\Psi=N e^{i S_{c l}\left[x_{c l}\right] / \hbar} \int e^{\int \frac{1}{2} \delta S_{c l}[x] / \delta x_{1} \delta x_{2} \mathcal{D} x_{1} \mathcal{D} x_{2}+\cdots} \mathcal{D} x=\sum_{n=0}(-i \hbar)^{n} \Psi_{n} e^{i S_{c l} / \hbar}
$$

with $x_{c l}$ the classical trajectory. This expression is known as the WKB expansion. For any group representation, this expansion means that:

$$
S[\Psi]=S_{c l}[x]+\mathcal{O}(\hbar)
$$

So, the $\hbar=0$ limit of $S[\Psi]$ in a first quantization scheme leads to $S_{c l}[x]$, in the same way as the $\hbar=0$ limit of the effective action conduces, in a second quantization scheme, to $S[\Psi]$.

We are interested in analyzing the case of a complex scalar field $\Phi$. Its action reads:

$$
S[\Psi]=\int \mathcal{L}(\Phi, \partial \Phi) d^{4} x=\int \frac{1}{2}\left(\partial^{\mu} \Phi \partial_{\mu} \Phi^{*}+\frac{m^{2}}{\hbar^{2}} \Phi \Phi^{*}\right) d^{4} x
$$

The corresponding energy-momentum tensor is:

$$
T^{\mu \nu} \equiv \frac{\partial \mathcal{L}}{\partial \partial_{\mu} \Phi} \partial^{\nu} \Phi+\frac{\partial \mathcal{L}}{\partial \partial_{\mu} \Phi^{*}} \partial^{\nu} \Phi^{*}-\mathcal{L} \eta^{\mu \nu}
$$

which conduces to:

$$
T^{\mu \nu}=\partial^{(\mu} \Phi \partial^{\nu)} \Phi^{*}-\frac{1}{2} \eta^{\mu \nu} \partial^{\lambda} \Phi \partial_{\lambda} \Phi^{*}-\frac{1}{2} \eta^{\mu \nu} \frac{m^{2}}{\hbar^{2}} \Phi \Phi^{*}
$$

The WKB expansion (3) for the scalar field reads:

$$
\Phi=\sum_{n=0}(-i \hbar)^{n} \varphi_{n} e^{i S_{c l} / \hbar}
$$

Now, using expansion (8) in expression (7), and after a straightforward computation, we find that up to order $1 / \hbar$, the energy momentum tensor can be written as:

$$
\begin{gathered}
T^{\mu \nu}=\frac{1}{\hbar^{2}} \partial^{\mu} S \partial^{\nu} S \varphi_{o} \varphi_{o}^{*}+\frac{1}{\hbar}\left\{\partial^{(\mu} S \operatorname{Im}\left(\varphi_{o} \partial^{\nu} \varphi_{o}^{*}\right)-2 \partial^{\mu} S \partial^{\nu} S \operatorname{Im}\left(\varphi_{o} \varphi_{1}^{*}\right)+\right. \\
\left.\eta^{\mu \nu}\left[\left(\partial^{\lambda} S \partial_{\lambda} S\right) \operatorname{Im}\left(\varphi_{o} \varphi_{1}^{*}\right)+\left(\partial^{\lambda} S\right) \operatorname{Im}\left(\varphi_{o} \partial_{\lambda} \varphi_{o}^{*}\right)+m^{2} \operatorname{Im}\left(\varphi_{o} \varphi_{1}^{*}\right)\right]\right\}
\end{gathered}
$$

where $S_{c l}=S$, and Im means "imaginary part". It is easy to verify that this symmetric quantity is conserved, i.e. satisfies:

$$
\partial_{\mu} T^{\mu \nu}=0
$$




\section{THE SCALAR FIELD AS A PERFECT FLUID}

Let us analyze the $\hbar^{-2}$ order (the first term) of expression (9). We may write the corresponding term as

$$
T^{\left(1 / \hbar^{2}\right) \mu \nu}=\hat{\rho} u^{\mu} u^{\nu}
$$

where

$$
\hat{\rho}=\frac{m^{2}}{\hbar^{2}} \varphi_{o} \varphi_{o}^{*}
$$

is the proper energy and $u^{\mu}$ is the timelike vector orthogonal to the hypersurface of constant phase $S$, where $\partial_{\mu} S=P^{\mu}$ and $S$ is understood as the Hamilton principal function. In the free case, canonical momentum $P^{\mu}$ coincides with the ordinary momentum $p^{\mu}$, i.e.:

$$
P^{\mu}=p^{\mu}=m u^{\mu}
$$

Four-velocity $u^{\mu}$ satisfies $u^{\mu} u_{\mu}=-1$. Relation (11) shows that $T^{\left(1 / \hbar^{2}\right) \mu \nu}$ is nothing but the energymomentum tensor of a dust.

Using (13) in (9) we are able to write the energy-momentum tensor showing explicitly the four-velocity components:

$$
\begin{aligned}
T^{\mu \nu}= & \frac{m^{2}}{\hbar^{2}} \varphi_{o} \varphi_{o}^{*} u^{\mu} u^{\nu}-2 \frac{m}{\hbar} u^{(\mu} \operatorname{Im}\left(\varphi_{o} \partial^{\nu)} \varphi_{o}^{*}\right) \\
& -2 \frac{m^{2}}{\hbar} u^{\mu} u^{\nu} \operatorname{Im}\left(\varphi_{o} \varphi_{1}^{*}\right)-\frac{m}{\hbar} u^{\lambda} \operatorname{Im}\left(\partial_{\lambda} \varphi_{o} \cdot \varphi_{o}^{*}\right) \eta^{\mu \nu}
\end{aligned}
$$

From (14) we can see that at a point where the observer is at rest (proper observer), i.e.: $\tilde{u}^{\mu}=$ $(-1,0,0,0)$, the components of $T^{\mu \nu}$, which we denote as $\tilde{T}^{\mu \nu}$, are:

$$
\begin{gathered}
\tilde{T}^{o o}=\frac{m^{2}}{\hbar^{2}} \varphi_{o} \varphi_{o}^{*}-\frac{m}{\hbar}\left[2 m \operatorname{Im}\left(\varphi_{o} \varphi_{1}^{*}\right)+\operatorname{Im}\left(\varphi_{o} \dot{\varphi}_{o}^{*}\right)\right] \\
\tilde{T}^{o i}=0 \\
\tilde{T}^{i j}=\frac{m}{\hbar} \operatorname{Im}\left(\varphi_{o} \dot{\varphi}_{o}^{*}\right) \delta^{i j}
\end{gathered}
$$

(with $i, j, \ldots=1,2,3$ ). This will be useful in order to find the general expression in any Lorentzian frame.

Once we have written the components of $T^{\mu \nu}$, we see that it is possible to define:

$$
\begin{gathered}
\rho=\frac{m^{2}}{\hbar^{2}} \varphi_{o} \varphi_{o}^{*}-\frac{m}{\hbar}\left[2 m \operatorname{Im}\left(\varphi_{o} \varphi_{1}^{*}\right)+\operatorname{Im}\left(\varphi_{o} \dot{\varphi}_{o}^{*}\right)\right] \equiv \hat{\rho}-\breve{\rho} \\
p=\frac{m}{\hbar} \operatorname{Im}\left(\varphi_{o} \dot{\varphi}_{o}^{*}\right)
\end{gathered}
$$

which are the proper energy density and the pressure, respectively. Here $\equiv d / d \tau, \tau$ being the proper time, $\hat{\rho}$ is given by (12) while $\breve{\rho}$ is $\breve{\rho}=\frac{m}{\hbar}\left[2 m \operatorname{Im}\left(\varphi_{o} \varphi_{1}^{*}\right)+\operatorname{Im}\left(\varphi_{o} \dot{\varphi}_{o}^{*}\right)\right]$. So, the components of $\tilde{T}^{\mu \nu}$ read:

$$
\begin{gathered}
\tilde{T}^{o o}=\rho \\
\tilde{T}^{o i}=0 \\
\tilde{T}^{i j}=p \delta^{i j}
\end{gathered}
$$


fluid.

Expressions (20-22) represent the fluid that a proper observer would describe as isotropic, i.e. a perfect

Now we want to generalize expressions (20-22) from this system to any Lorentz frame. To do this we analyze how $T^{\mu \nu}$ may be 'measured' in a reference frame at rest in the laboratory supposing that the fluid appears to be moving at each space-time point with velocity $v^{i}$. This velocity is related to the spatial components of the four velocity $u^{i}$ of the fluid via:

$$
v^{i}=\gamma^{-1} \cdot u^{i}
$$

where $\gamma=\left(1-\bar{v}^{2} / c^{2}\right)^{-1 / 2}$ is the boost that transforms Lorentz reference frames among them. Applying this boost to components $(20-22)$ of $T^{\mu \nu}$, we obtain:

$$
\begin{gathered}
T^{o o}=\left(\rho+p \bar{v}^{2}\right) \gamma^{2} \\
T^{o i}=(\rho+p) v^{i} \gamma^{2} \\
T^{i j}=p \delta^{i j}+(\rho+p) v^{i} v^{j} \gamma^{2}
\end{gathered}
$$

with $\rho$ and $p$ given by (18) and (19), respectively.

Combining (23), (24) and (25), we see that in any Lorentz frame, expression (14) reads:

$$
T^{\mu \nu}=\rho u^{\mu} u^{\nu}+p\left(\eta^{\mu \nu}+u^{\mu} u^{\nu}\right)
$$

i.e.: a perfect fluid. In conclusion: expression (9) for $T^{\mu \nu}$ which is given by QFT, corresponds to a perfect fluid that can be written only in terms of macroscopical variables.

In order to connect proper energy density and pressure we would only need an equation of state. The structure of this equation depends on the properties of the system. In the particular case in which temperature is an independent variable, pressure is a function of $\rho_{o}$ and $\Pi$ :

$$
p=p\left(\rho_{o}, \Pi\right)
$$

where

$$
\rho=\rho_{o}(1+\Pi)
$$

$\rho_{o}$ being the proper mass density and the product $\rho_{o} . \Pi$, the internal energy density.

\section{RELATION BETWEEN THE WKB AMPLITUDES AND EQUATIONS OF MOTION}

In QFT, field equations are obtained minimizing the effective action with respect to the field (equation (2)). For the scalar representation $\Phi$, this conduces to the Klein-Gordon equation:

$$
\square \Phi+\frac{m^{2}}{\hbar^{2}} \Phi=0
$$

which in terms of the WKB expansion of the field, reads:

$$
\left.\sum_{n}\left[\hbar^{2} \square \phi_{n}+i \hbar\left(2 \partial^{\mu} \phi_{n} \partial_{\mu} S+\phi_{n} \square S\right)-\phi_{n} \partial^{\mu} S \partial_{\mu} S\right)(-i \hbar)^{n}\right]=-\sum_{n} m^{2} \phi_{n}(-i \hbar)^{n}
$$

Assuming that (30) must be satisfied order by order in $\hbar$, we obtain the differential equations which are needed to describe the perfect fluid via eqs. (18) and (19):

$$
\begin{array}{cc} 
& n=0 \\
n=1 & 2 i \partial^{\mu} \phi_{o} \partial_{\mu} S+i \partial_{\mu} S+m^{2}=0 \\
n &
\end{array}
$$

Equation (31) provides no new information: it is trivially satisfied because $\partial^{\mu} S=P^{\mu}=m u^{\mu}$ and $u^{\mu} u_{\mu}=-1$. Equation (32) may be written as:

$$
2 u^{\mu} \partial_{\mu} \phi_{o}+\left(\phi_{o}-i \phi_{1}\right) \partial_{\mu} u^{\mu}-i m \phi_{1}=0
$$

and relates the first two amplitudes of the WKB expansion of the field. 
In order to find the equations of motion corresponding to scalar particles, we compute equation (10) using (19) and obtain:

$$
\partial^{\mu} P+\partial_{\nu}\left[(\rho+P) u^{\mu} u^{\nu}\right]=0
$$

Keeping only those equations corresponding to the spatial components of the velocity $\bar{v}$, we find the following vectorial equation:

$$
(\rho+P) \cdot\left[\frac{\partial \bar{v}}{\partial t}+(\bar{v} \cdot \bar{\nabla}) \bar{v}\right]=-\gamma^{-2}\left[\bar{\nabla} P+\bar{v} \frac{\partial P}{\partial t}\right]
$$

where we have used the fact that $u^{i}=v^{i} \cdot u^{o}$. In eq. (35), different orders in $1 / \hbar^{n}$ are involved due to the corresponding expressions of $\rho$ and $P$ (see (18) and (19)). So, for the first two orders, we obtain two equalities. Up to the $1 / \hbar^{2}$ (main) contribution we have :

$$
\frac{\partial \bar{v}}{\partial t}+(\bar{v} \cdot \bar{\nabla}) \bar{v}=0
$$

Taking into account the $1 / \hbar$ correction, eq. (36) becomes:

$$
(\hat{\rho}-\breve{\rho}+P) \cdot\left[\frac{\partial \bar{v}}{\partial t}+(\bar{v} \cdot \bar{\nabla}) \bar{v}\right]=-\gamma^{-2}\left[\bar{\nabla} P+\bar{v} \frac{\partial P}{\partial t}\right]
$$

The first equation tells us that, at the highest order in the WKB expansion, the free massive scalar field is represented by particles with null acceleration, i.e., their trajectories are straight lines. This is the classical limit of the approach and represents the inertial principle. But we can see from eq. (37) that quantum corrections to the straight line appear at the next order. That is to say: free matter follows trajectories that deviate from straight lines and from non-accelerated uniform motion.

We want to recall that equation (37) may be considered the relativistic second law of Newton: $F^{\mu}=m \cdot d u^{\mu} / d \tau$, where quantum corrections to $\rho$ and the appearance of $P$ (expressions (18) and (19) respectively), are responsible for deviations from straight lines with no need of external forces nor of quantum potentials (as, for example, in the Bohm's scheme; see[4]). Equation (37) reveals the nature of the internal structure of matter and how it acts in order to produce quantum effects.

\section{SUMMARY}

We have developed the WKB expansion of the scalar field up to the first quantum correction and studied the relations between QFT description of matter and macroscopic fluids. Our aim was to find the behaviour of this matter in a macroscopical picture, asumming quantum corrections. Our main results are the following:

- at the first quantum approximation, free scalar fields correspond to perfect fluids with pressure,

- the corresponding law of motion implies deviations from straight unaccelerated motion.

\section{REFERENCES}

[1] M. Castagnino, G. Domenech, M. Levinas and N. Umérez, Class.Quant.Grav. 6 (1989) L 1.

[2] G. Domenech, M. Levinas and N. Umérez, Phys. Lett. 137A (1989) 17.

[3] R. Feynman and A. Hibbs, Quantum Mechanics and Path Integral, New York, Mc.Graw-Hill, 1965, chap. 4.

[4] D. Bohm, B. Hiley and P. Kaloyerou, Phys. Rep.144 No. 6 (1987) 321. 